\title{
Habitability of Brown Dwarf Planets
}

\author{
Andrey Andreeshchev and John Scalo \\ Department of Astronomy, University of Texas, Austin TX 78712, \\ U.S.A
}

\begin{abstract}
The time during which the temperature of a terrestrial-like planet remains in the liquid water range during the fading of its parent brown dwarf is calculated as a function of brown dwarf mass and planetary semimajor axis using recent models for brown dwarf evolution and two criteria for habitable zone width. Durations of habitability range from 0.5-2 Gyr at a brown dwarf mass of $0.03 \mathrm{M}_{\odot}$ to 2-10 Gyr at a brown dwarf mass of $0.07 \mathrm{M}_{\odot}$ for planets within a few Roche radii.
\end{abstract}

\section{Introduction}

Brown dwarfs (BDs) are objects whose masses are below the limit required for hydrogen fusion reactions to occur before core electron degeneracy sets in, about $0.077 \mathrm{M}_{\odot}$ for solar metallicity (see Burrows et al. 1997). Their evolution consists of continual fading as gravitational potential energy is released, on timescales that depend on the $\mathrm{BD}$ mass and age. Recent censuses of both field stars and open clusters indicate that the number of brown dwarfs is comparable to the number of low-mass main sequence stars, which constitute the majority of stars (e.g. Chabrier 2002). Several lines of evidence suggest that planets may form in orbit around BDs: detection of a planet orbiting the M star Gl 876 (Delfosse et al. 1998; Marcy et al. 1998), the existence of analogous satellite systems around giant planets in our solar system, the logarithmic succession of planetary semimajor axes in our solar system extended to lower-mass stars, simulations that indicate terrestrial-mass habitable zone planets can form around low-mass objects as long as disk material of sufficient surface density is originally placed close enough to the parent star (Wetherill 1996), and indications from nearinfrared excess emission that BDs in several young regions possess disks (e.g., Muench et al. 2001; Haisch et al. 2001; Testi et al. 2002).

\section{Habitable Zone Radii}

The origin of terrestrial life required at least $0.5 \mathrm{Gyr}$ and complex multicellular life perhaps $3 \mathrm{Gyr}$, so we consider the conditions under which a terrestrial-mass planet orbiting a fading $\mathrm{BD}$ can remain habitable. We adopt a conventional surface liquid water definition of "habitable zone" (HZ). Following Kasting et al. (1993) we adopt fluxes at the inner and outer edges of the HZ corresponding to an inner radius of $0.95 \mathrm{AU}$ and an outer radius of $1.4 \mathrm{AU}$ for the solar system. The inner radius denotes "moist stratosphere" limit, while the outer limit is the 
distance of maximum possible $\mathrm{CO}_{2}$ greenhouse heating. The corresponding flux in units of the solar constant $S_{e}$ are 1.1-0.51. We recognize the significant uncertainty in these values, especially as applied to BD planets, and give some results for a second, less conservative, choice of boundaries. In this case, the inner and outer edges correspond to the irradiance at $0.84 \mathrm{AU}$ and $2 \mathrm{AU}$ in the solar system. The first value marks where "runaway greenhouse" is expected, and the second one is a compromise between the conservative 1.4 AU limit and 2.4 AU distance of the maximum warming due to complete $\mathrm{CO}_{2}$ ice cloud cover (Forget \& Pierrehumbert 1997; Mischna et al. 2000). In this case the corresponding flux limits are $1.4 \mathrm{~S}_{\mathrm{e}}-0.25 \mathrm{~S}_{\mathrm{e}}$.

We use the BD evolutionary models of Burrows et al. (1997), supplemented and checked with the models of Chabrier et al. (2000), to cover the range of masses from $0.02 \mathrm{M}_{\odot}-0.2 \mathrm{M}_{\odot}$. As a $\mathrm{BD}$ of given mass fades, the habitable zone moves past a planet located at a given distance from the $\mathrm{BD}$, yielding the duration of habitability. Figure 1 shows the habitable distances for $1 S_{e}$ as a function of mass for three times. The habitable zone is well within the tidal lock radius (Kasting et al. 1993) for synchronous rotation even for young BDs, but, following the work of Joshi et al. (1997), there is no longer reason to suppose that atmospheric freeze-out will occur even if the atmosphere is much thinner than the Earth's. The Roche limit for a planet with a density equal to that of the Earth is also shown, since there should be no planets within this radius.

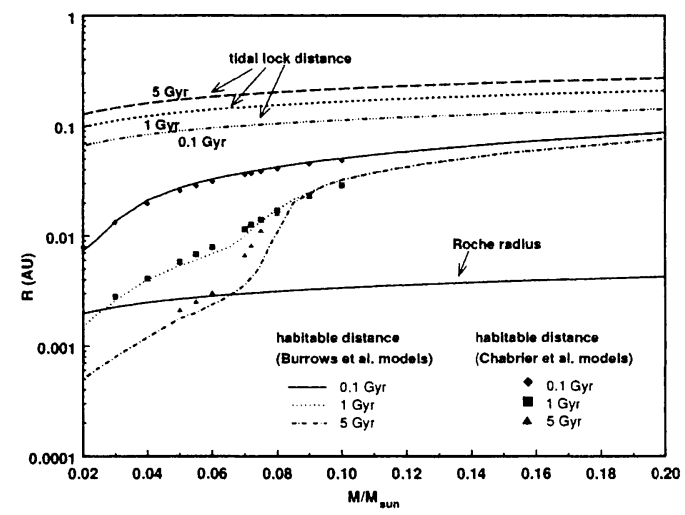

Figure 1. Habitable distance (for $1 \mathrm{~S}_{\mathrm{e}}$ flux), tidal lock distance, and Roche radius as a function of mass of parent star or brown dwarf for 3 different ages.

\section{Duration of Habitable Zone Residence}

Our central question is whether a planet at some given distance from a BD will remain in the shrinking $\mathrm{HZ}$ long enough for life, or advanced life forms, to develop. Based on Earth as our only example, we might expect that a timescale of habitability of order a billion years is required for life, and especially advanced life. Given that the mutational environment of putative organisms on a brown dwarf planet should be very different from Earth, it is clear that evolutionary 
timescales could be much different. Yet if the calculated habitability duration for $\mathrm{BD}$ planets were less than $0.1 \mathrm{Gyr}$, say, one might seriously question the viability of the development of complex life on such planets. The only earlier such calculations we know of are by Desidera (1999) who considered planets at different assumed distances from a $0.04 \mathrm{M}_{\odot}$ BD (meant to model Gl 229B), finding durations up to $1 \mathrm{Gyr}$ for a planet near the tidal destruction radius. The present results show how the duration depends on the BD mass, as well as its sensitivity to the adopted $\mathrm{HZ}$ limits.

Figure 2 shows the calculated duration of residence in the evolving $\mathrm{HZ}$ for planets as a function of the the semimajor axis a of the planet's orbit, assumed circular, for various BD masses. The solid lines show the results for a conservative choice of $\mathrm{HZ}$ limits which correspond to $0.95 \mathrm{AU}$ and $1.4 \mathrm{AU}$ for the solar system, while the dashed lines show results for a more liberal choice of 0.84 to 2.0 AU. Semimajor axes below the Roche limit (crosses) are not allowed. The strong dependence on choice of $\mathrm{HZ}$ limits is evident, but in either case it is clear that habitability durations of order $1 \mathrm{Gyr}$ are possible for planets within 2-3 Roche radii for $\mathrm{BD}$ masses above $0.03 \mathrm{M}_{\odot}$. $\mathrm{HZ}$ durations as long as $4 \mathrm{Gyr}$ are only possible close to the $\mathrm{BD}$ limit in the conservative case, but can occur for BDs as small as $0.04 \mathrm{M}_{\odot}$ if the planet is sufficiently close to the Roche limit. Given the uncertainties, upper limits for the duration of habitability (i.e. for planets at the Roche limit) range from $1-2 \mathrm{Gyr}$ at a BD mass of $0.03 \mathrm{M}_{\odot}$ to 4-10 Gyr at a BD mass of $0.07 \mathrm{M}_{\odot}$.

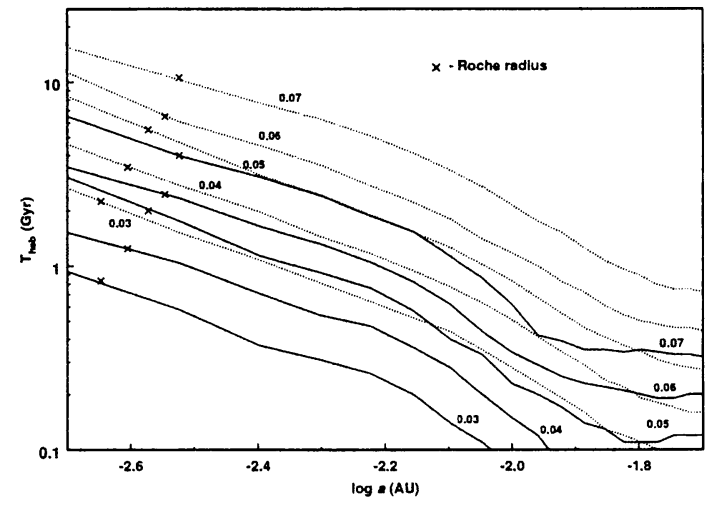

Figure 2. Duration of liquid water habitability for planets orbiting brown dwarfs of various masses as a function of semimajor axis of planet. Solid lines and dotted lines correspond to conservative and liberal estimates of habitable zone extent.

\section{Additional Questions}

Other issues of interest include: 1) whether solid planets can survive sublimation during the phase when the BD is bright if their semimajor axes are small enough to result in large durations of habitability; 2) whether "hot start" initial conditions would lead to atmospheric loss before clement temperatures are reached; 3 ) the gradual decrease in average planetary temperature by a factor of 
about $2 / 3$ as the $\mathrm{HZ}$ passes by a BD planet-extremophiles suggest adaptability can occur, but nucleic acid thermal stability may be a problem; however, various climatological effects, especially those that use $\mathrm{CO}_{2}$ feedback (e.g. Kasting et al. 1993; Sleep \& Zahnle 2001) should moderate the temperature decrease; 4) irradiance fluctuations due to irregular BD cloud cover and flare activity; 5) photosynthesis (Heath et al. 1999; Wolstencroft \& Raven 2000) - very little flux for terrestrial-like photosystems, but $\mathrm{CO}_{2}$ levels increasing with time due to decreasing weathering; and 6) if development of intelligence is partially driven by cooling episodes, as suggested by Schwartzman \& Middendorf (2000), then on $\mathrm{BD}$ planets cognitive evolution may be expected to contain a stronger continuous component than on Earth. We postpone further discussion of these questions to future work.

Acknowledgments. We thank Jim Kasting for useful communications. This work was supported by NSF grant 9907582 .

\section{References}

Burrows, A., et al. 1997, ApJ, 491, 856

Chabrier, G., Baraffe, I., Allard, F. \& Hauschildt, P. H. 2000, ApJ, 542, 464

Chabrier, G. 2002, ApJ, 567, 304

Delfosse, X., Forveille, T., Mayor, M., Perrier, C., Naef, D., \& Queloz, D. 1998, A\&Ap, 338, 67

Desidera, S. 1999, PASP, 111, 1529

Forget, F., \& Pierrehumbert, R. T. 1997, Science, 278, 1273

Haisch, K. E., Lada, E. A., \& Lada, C. J. 2001, AJ, 121, 2065

Heath, M. J., Doyle, L. R., Joshi, M. M., \& Haberle, R. M. 1999, 29, 405

Joshi, M. M., Haberle, R. M., \& Reynolds, R. T. 1997, Icarus, 129, 450

Kasting, J. F., Whitmire, D. P. \& Reynolds, R. T. 1993, Icarus, 101, 108

Marcy, G., Butler, P., Vogt, S., Fischer, D., \& Lissauer, J. J. 1998, ApJ, 505, L147

Mischna, M. A., Kasting, J. F., Pavlov, A. \& Freedman, R. 2000, Icarus, 145, 546

Muench, A. A., Alves, J., Lada, C.J. \& Lada, E.A. 2001, ApJ, 558, L51

Schwartzman, D., \& Middendorf, G. 2000, in ASP Conf. Ser. 213, Bioastronomy '99: A New Era in the Search for Life, ed. G. Lemarchand \& K. Meech, 425

Sleep, N. H., \& Zahnle, K. 2001, J. Geophys. Res., 106, 1373

Testi, L., et al. 2002, ApJ, 571, L155

Wetherill, G. W. 1996, Icarus, 119, 219

Wolstencroft, R. D., \& Raven, J. A. 2000, in ASP Conf. Ser. 213, Bioastronomy '99: A New Era in the Search for Life, ed. G. Lemarchand \& K. Meech, 343 